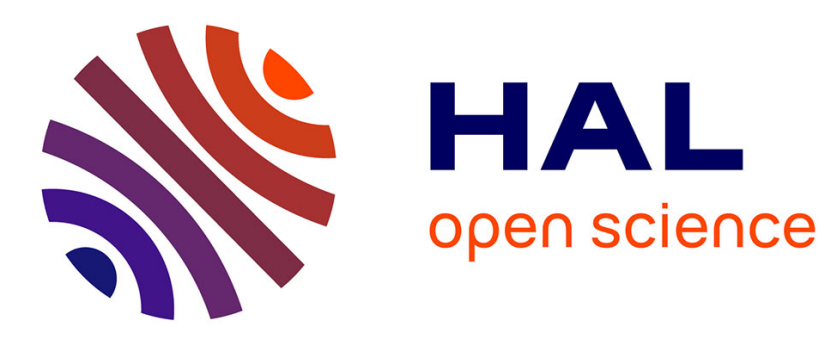

\title{
Tentative detection of clear-air turbulence using a ground-based Rayleigh lidar
}

Alain Hauchecorne, Charles Cot, Francis Dalaudier, Jacques Porteneuve, Thierry Gaudo, Richard Wilson, Claire Cénac, Christian Laqui, Philippe

Keckhut, Jean-Marie Perrin, et al.

\section{To cite this version:}

Alain Hauchecorne, Charles Cot, Francis Dalaudier, Jacques Porteneuve, Thierry Gaudo, et al.. Tentative detection of clear-air turbulence using a ground-based Rayleigh lidar. Applied optics, 2016, 55 (13), pp.3420-3428. 10.1364/AO.55.003420 . insu-01322228

\section{HAL Id: insu-01322228 \\ https://hal-insu.archives-ouvertes.fr/insu-01322228}

Submitted on 30 May 2016

HAL is a multi-disciplinary open access archive for the deposit and dissemination of scientific research documents, whether they are published or not. The documents may come from teaching and research institutions in France or abroad, or from public or private research centers.
L'archive ouverte pluridisciplinaire HAL, est destinée au dépôt et à la diffusion de documents scientifiques de niveau recherche, publiés ou non, émanant des établissements d'enseignement et de recherche français ou étrangers, des laboratoires publics ou privés. 


\title{
Tentative detection of clear air turbulence using a ground-based Rayleigh lidar
}

\author{
Alain HaucheCorne ${ }^{1}$, Charles Cot $^{1}$, Francis Dalaudier ${ }^{1}$, JACQues

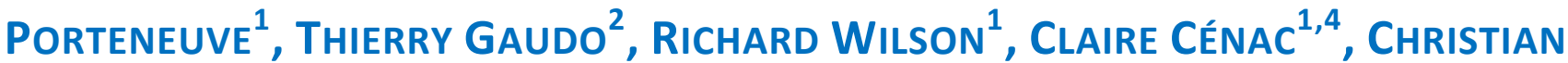

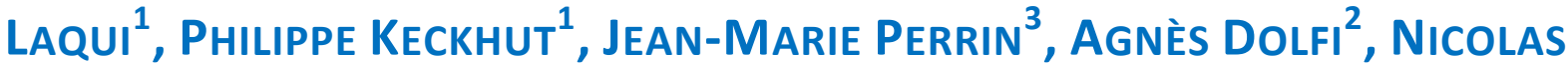 \\ CÉZARD $^{2}$, LAURENT LOMBARD ${ }^{2}$, CLAUdine BESSON ${ }^{2}$ \\ ${ }^{1}$ LATMOS/IPSL, UVSQ Université Paris-Saclay, UPMC Univ. Paris 06, CNRS, Guyancourt, France \\ ${ }^{2}$ ONERA/DOTA, Palaiseau, France \\ ${ }^{3} O H P, C N R S-I N S U$, Saint-Michel I'Observatoire, France \\ ${ }^{4}$ Currently with Laboratoire de Météorologie Dynamique, Ecole Polytechnique, Palaiseau, France \\ *Corresponding author: alain.hauchecorne@latmos.ipsl.fr
}

Received XX Month XXXX; revised XX Month, XXXX; accepted XX Month XXXX; posted XX Month XXXX (Doc. ID XXXXX); published XX Month XXXX

\begin{abstract}
Atmospheric gravity waves (GWs) and turbulence generate small-scale fluctuations of wind, pressure, density and temperature in the atmosphere. These fluctuations represent a real hazard for commercial aircraft and are known as the generic name of Clear Air Turbulence (CAT). Numerical Weather Prediction models do not resolve CAT and therefore provide only a probability of occurrence. A ground-based Rayleigh lidar was designed and implemented to remotely detect and characterize the atmospheric variability induced by turbulence in vertical scales between $40 \mathrm{~m}$ and a few $100 \mathrm{~m}$. Field measurements were performed at Observatoire de Haute-Provence (OHP, France) in December 8, 2008 and June 23, 2009. The estimate of the mean squared amplitude of bidimensional fluctuations of lidar signal showed excess compared to the estimated contribution of the instrumental noise. This excess can be attributed to atmospheric turbulence with a $\mathbf{9 5 \%}$ confidence level. During the first night, data from collocated ST (Stratosphere-Troposphere) radar were available. Altitudes of the turbulent layers detected by the lidar were roughly consistent with those of layers with enhanced radar echo. The derived values of turbulence parameters $C_{n}{ }^{2}$ or $C_{T}{ }^{2}$ were in the range of those published in the literature using ST radar data. However the detection was at the limit of the instrumental noise and additional measurement campaigns are highly desirable to confirm these initial results. This is to our knowledge the first successful attempt to detect CAT in the free troposphere using an incoherent Rayleigh lidar system. The built lidar device may serve as a test bed for the definition of embarked CAT detection lidar systems aboard airliners.
\end{abstract}

OCIS codes: (010.0010) Atmospheric and oceanic optics; (010.3640) Lidars; (010.7060) Turbulence.

http://dx.doi.org/10.1364/AO.99.099999

\section{INTRODUCTION}

Clear Air Turbulence (CAT) refers to fluctuations with time scales ranging from a few seconds to a few ten seconds and spatial scales ranging from a few ten meters to a few hundred meters, due to turbulence and gravity waves (GWs) in air without cloud, usually located in high troposphere and low stratosphere. Those CAT are a serious issue for in-flight plane security and are responsible for numerous injuries or even reported deaths every year [1-2]. Planes crossing such CAT can be strongly shaken and passengers with unfastened security belt can be seriously injured. There is nowadays no alert system able to detect CAT at typical flight height. Indeed, onboard weather radars are blind to clear air turbulence. Alert notifications result most of the time from phone communications between airplanes on the same airline route.

Wind and temperature fluctuations in the lower atmosphere can be described over a wide range of scales. In the mesoscale range from a few ten meters to some kilometers along the vertical and a few hundred meters to a few hundred kilometers along the horizontal, these fluctuations result mainly from a GW field. A wide variety of observational techniques have been used to study mesoscale fluctuations in the atmosphere, one will quote in particular balloon soundings and aircraft observations for in-situ techniques [3-6], radar observations and lidar studies for remote sensing techniques [7-11]. A common feature of these experimental studies is an approximately invariant "universal" vertical and frequency power spectrum, regardless of season and geographic location. This feature, first recognized by [12] who took as starting point oceanographic experimental studies [13], led to the concept of a "universal" spectrum of atmospheric fluctuations constrained to remain with the same shape and same level in the spectral domain everywhere in the world except near GWs sources. Several saturation theories have since emerged. Each of them proposes a physical mechanism thought to be responsible to limit wave amplitude and each predicts with a good 
approximation shape and level of vertical wave number power spectrum.

Turbulence is associated to this wave field, generated when the wave field saturates and then breaks. It can result from breakdown of stratified shear layers (Kelvin-Helmholtz instability). Turbulence is a chaotic non-linear superposition of rotational movement. These vortices, generated by instabilities, decay into smaller vortices until the vorticity is finally dissipated by viscosity.

Today, CAT is a serious flight security problem because there exist no ground or onboard detection devices. Although the CAT phenomenon is thought to be due to dynamics instabilities and waves in the atmosphere [14], the physical phenomenon is far from being clearly understood. The understanding of CAT phenomenon is poor because: 1) it is difficult to measure the CAT atmospheric parameters in situ (relatively low occurrence); 2) the simulations are complex. Therefore, this original project intended to obtain a comprehensive measured data set by remote sensing from a ground-based lidar.

For aeronautic applications CAT is classified according to the vertical acceleration experienced by the airplane. It is due firstly to the vertical wind but also to the fluctuations in atmospheric density and horizontal wind. CAT is producing fluctuations in all atmospheric parameters characterizing the state of the atmosphere: atmospheric density, temperature and the three components of the wind. In principle it is possible to detect a turbulent layer by measuring only one of these parameters. However the relative amplitudes of the fluctuations are related by relations involving the stability of the atmosphere characterized by the Brunt-Väisälä frequency $N$. In the domain of isotropic turbulence the fluctuations of the three components of the wind have the same amplitude. Fluctuations in density and temperature are induced by the vertical displacement of air parcels having a potential temperature different from the surrounding air. They are then related to the vertical gradient of potential temperature. In an atmospheric layer with adiabatic lapse rate $(N=0)$, fluctuations in the vertical wind will have no effect on the density and temperature fluctuations. On the contrary, in an atmospheric layer with a high stability, vertical wind fluctuations induce large density and temperature fluctuations. It is therefore not equivalent to try to detect turbulent layers using wind or density/temperature measurements.

Different techniques have been proposed and developed to detect the atmospheric turbulence by lidar. The most developed one is the coherent Doppler lidar technique to observe vertical and horizontal wind fluctuations. This technique requires a high signal to noise ratio on aerosol scattering and is mainly used in the atmospheric boundary layer [15-16]. A CAT layer was detected at $12 \mathrm{~km}$ altitude with an airborne coherent lidar developed by JAXA (Japanese Aerospace Exploration Agency) [17]. However, the efficiency of the coherent lidar is strongly depending on the aerosol loading which may be very low at the flight level of commercial aircraft and is therefore not well adapted for an operational use. Another proposed technique is the detection of density fluctuations from the measurement of Rayleigh scattering with an incoherent lidar. The difficulty of this technique is the separation between the Rayleigh signal due to molecular scattering proportional to the atmospheric density and the signal due to aerosol backscattering. The best way to do that is to use a high-spectral resolution lidar (HSRL) able to separate the Doppler broadened molecular scattering from the narrow spectral aerosol scattering. The spectral separation can be made with a Fabry-Pérot etalon [18], a narrow band iodine filter [19-21] or a Fizeau interferometer in the ALADIN lidar on board the space wind lidar ADM-Aeolus [22]. Published results using HSRLs concern up to now observations of aerosols and wind. Such method was also proposed to be implemented in an airborne lidar for the detection of CAT in the frame of the
European FP7 project DELICAT (Demonstration of Lidar based Clear Air Turbulence detection) [23]. Finally [24] proposed a detection of turbulence based on the backscatter enhancement effect when the backscattered light propagates exactly in the same inhomogeneities than the emitted laser beam (laser emission coaxial with the receiving telescope). This new promising method has been only studied theoretically.

Although short-scale GWs contribute to CAT, we will concentrate in this article on turbulence that is the most active phenomenon to induce fluctuations on atmospheric density and temperature at scales shorter than a few hundred meters and can be retrieved from the molecular backscattering by use of an incoherent Rayleigh lidar set-up at Haute-Provence Observatory (OHP, $44^{\circ} \mathrm{N}, 6^{\circ} \mathrm{E}$ ). This lidar is not equipped with a high-resolution spectral filter for the separation between molecular and aerosol scattering. Since solid aerosols depolarize the backscattered light, a polarization beam-splitter cube is used to detect the presence of aerosol layers to prevent the use of data contaminated by fluctuations of aerosol scattering. The lidar returns passing through the polarization discriminator can be processed to retrieve turbulence profiles when the contribution of aerosol scattering to the total signal is sufficiently low. It is not possible to ensure that the atmosphere is totally free from aerosols. However, the CAT detection is based on the estimation of relative fluctuations of the backscatter signal, assumed to be not modified in presence of low-level scattering due to a well-mixed aerosol layer, the ratio between aerosol and molecular backscattering remaining constant inside this layer.

In the second section we present the principle of CAT detection using the average of the squared amplitude of fluctuations in the Rayleigh lidar signal, called hereafter $F Q$ for fluctuation quantity and we simulate the expected performances. In the third section we describe the instrumental setup at OHP and data processing for CAT detection and in the fourth section we discuss the results obtained during field campaigns showing the likely detection of CAT layers..

\section{EXPECTED PERFORMANCES FOR CAT DETECTION}

In a turbulent layer, atmospheric density presents 3D fluctuations at different scales. The amplitude of these fluctuations is related to the intensity of the turbulence and has a 3D spectrum depending on its nature. For an isotropic 3D turbulence field the Kolmogorov law predicts a $-5 / 3$ spectral slope in vertical and horizontal spectra [25]. It means that fluctuations are expected to be larger at larger scales.

A Rayleigh-Mie lidar is a system able to measure the vertical profile of light emitted by a pulsed visible or near UV laser and backscattered by atmospheric molecules and particles. Its signal is the sum of a Rayleigh scattering signal directly proportional to the molecular number density and a scattering signal depending on the number and the nature of atmospheric particles.

The lidar equation in elastic scattering mode (backscattering occurring at the wavelength of the laser, the Raman scattering is not considered here) is:

$S(z, z+d z)=E_{0} t_{\text {atm }}^{2}(0, z) Q_{l i d} \frac{A_{t e l}}{z^{2}}\left[\beta_{r}(z) n_{r}(z)+\beta_{m}(z) n_{m}(z)\right]$

where:

- $S(z, z+d z)$ is the measured signal backscattered in the altitude interval $[z, z+d z]$,

- $\quad E_{0}$ is the number of photons emitted by the laser,

- $\operatorname{tatm}(0, z)$ is the atmospheric transmission between the ground and the altitude $z$,

- $Q_{\text {lid }}$ is the optical efficiency of the lidar system, 
- $\quad A_{t e l}$ is the telescope area,

- $\quad \beta_{r}(z)$ and $\beta_{m}(z)$ are respectively the air molecules and aerosols backscattering cross sections at $z$,

- $\quad n_{r}(z)$ and $n_{m}(z)$ are respectively the air molecules and aerosols concentrations at $z$.

In general, these lidars are used to determine the vertical profile of atmospheric density in altitude ranges where air is free of aerosols, for instance above $30 \mathrm{~km}$ where it is possible to compute the vertical temperature profile from the vertical density profile [26]. For this application, the lidar signal can be integrated in time and in vertical to increase the signal to noise ratio (during a few minutes to several hours and over a few hundred meters to a few kilometers). In the present study our goal is to use a Rayleigh-Mie lidar to detect density fluctuations due to turbulence. For this purpose we can only use data when the Mie signal is negligible compared to the Rayleigh signal (less than about 5\%). This happens generally in the upper troposphere (5$10 \mathrm{~km}$ ) where air is dry enough to prevent the formation of clouds. In a lidar signal it is easy to detect the presence of cloud particles and to eliminate contaminated data. In this case it is necessary to record the signal with a time and vertical resolution adapted to the scale of turbulent structures (at least the largest ones). Assuming an isotropic turbulence layer with external scale equal or greater than $100 \mathrm{~m}$ and with the hypothesis that turbulent structures are advected by a $10 \mathrm{~ms}^{-1}$ mean wind, the lidar signal should be recorded with at least $50 \mathrm{~m}$ vertical resolution and $5 \mathrm{~s}$ time integration. This is feasible with modern lidar data acquisition systems but the signal to noise ratio for a single measurement will be too low to be exploited. To work around this problem, we propose to average the squared amplitude of density fluctuations $F Q$ instead of averaging the signal itself. In turbulent atmospheric layers, the mean amplitude of lidar signal fluctuations will slightly increase. This increase will not be detectable on a single profile integrated during a few seconds but will increase the $F Q$ averaged over a longer period (for instance 15 to 60 minutes). To do that it is needed to have an accurate estimate of the instrumental noise. It will not give the instantaneous amplitude of the turbulent perturbations but will provide their mean value during the period over which the $F Q$ is averaged. The way to estimate the instrumental noise is explained in the data processing section.

The expected signal with the OHP Rayleigh lidar is estimated using the instrumental and atmospheric parameters given in Tables 1 and 2 . The number of photo-electrons counted for $1 \mathrm{~s}$ time integration and 15 m vertical integration is $N_{p h}=36000$ at $10 \mathrm{~km}$ altitude.

The accuracy of the Rayleigh density lidar considering only the Poisson noise is given by:

$$
\frac{\Delta \rho}{\rho}=\frac{1}{\sqrt{N_{p h}}}
$$

We consider that an increase of the variance fluctuations indicates a significant contribution of atmospheric fluctuations if it exceeds the estimated instrumental variance by 2 standard deviations. Figure 1 presents this 2 sigma detectability limit (red curves) expected using $Q_{\text {lid }}=0.03$, which is a conservative value for 1 second and 1 minute time integration and in blue the expected amplitude of density fluctuations (blue curves) as a function of the external scale of turbulence assuming. The amplitude of density fluctuations is computed for a tropospheric and a stratospheric potential temperature gradient (Brunt-Väisälä frequency respectively $N=1 \cdot 10^{-2}$ and $2 \cdot 10^{-2} \mathrm{~s}^{-1}$ ). According to [27], for a given external scale of turbulence density and temperature fluctuations are proportional to $N^{2}$.:

$$
N^{2}=\frac{g}{\theta} \frac{d \theta}{d z}
$$

where $\theta$ is the potential temperature and $z$ the altitude.

Table 1. System Parameters for transmitter and receiver

\begin{tabular}{ll}
\hline Instrumental parameters & \\
\hline Laser & Pulsed Nd:YAG \\
Average power & $E_{0}=15 \mathrm{~W} @ 532 \mathrm{~nm}$ \\
Laser emission & $4.10^{19} \mathrm{ph} \mathrm{s}^{-1}$ \\
Pulse repetition rate & $50 \mathrm{~Hz}$ \\
Energy per pulse & $300 \mathrm{~mJ} @ 532 \mathrm{~nm}$ \\
Off axis & $6 \mathrm{~m}$ \\
Telescope & Newton \\
Focal & $1500 \mathrm{~mm}-$ \\
Diameter & $530 \mathrm{~mm}$ \\
Pupil surface & $\mathrm{A}_{\text {tel }}=0.22 \mathrm{~m}^{2}$ \\
Field of view & $0.67 \mathrm{mrad}$ \\
Multimode fiber & $1 \mathrm{~mm}-$ diameter \\
Interferential filter & $61 \%(B W=1 \mathrm{~nm})$ \\
Polarizer & $\mathrm{T}>95 \% \mathrm{R}>99 \%$ \\
Photomultiplier tubes & Hamamatsu-R7205-01 \\
Bandwidth & $3.5 \mathrm{MHz}$ \\
Quantum efficiency & $10 \%$ \\
Analogic signal Recorder & $\mathrm{NI} 5922$ \\
Sampling frequency & $10 \mathrm{MHz}$ \\
Overall lidar efficiency & $\mathrm{Q}_{\text {lid }}=0.1-0.01$ \\
Spatial resolution & $\mathrm{d}_{\mathrm{z}}=15 \mathrm{~m}$
\end{tabular}

Table 2. Atmospheric parameters Parameters

Altitude

$\mathrm{z}=10000 \mathrm{~m}$

Transmission (1 path)

$t_{\text {atm }}=0.7$

Molecular backscattering

Aerosol backscattering

$\beta_{R}=4 \cdot 10^{-7} \mathrm{~m}^{-1} \mathrm{sr}^{-1}$

Horizontal mean wind

$\beta_{\mathrm{M}}<0.05 \beta_{R}$

$10 \mathrm{~ms}^{-1}$

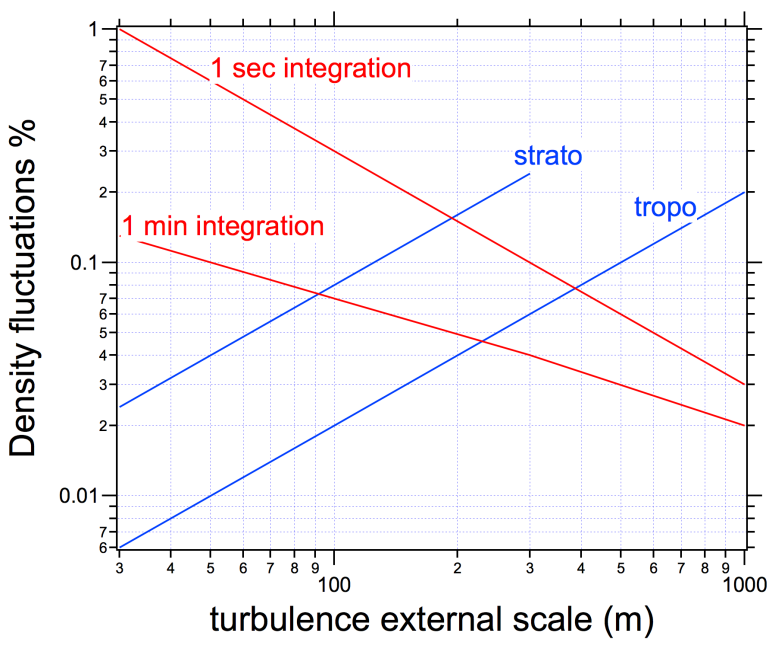

Figure 1: Estimated density fluctuations as a function of the turbulence external scale for stratospheric and tropospheric potential temperature gradients (blue curves) and 2- $\sigma$ detectability limit for $1 \mathrm{~s}$ and $1 \mathrm{~min}$ integration time (red curves). Density fluctuations are proportional to $N^{2}$ and to the sampling distance taken equal to thickness of the turbulence layer.

With these parameters we should be able to detect in one minute turbulent layers larger than $100 \mathrm{~m}$ in the stratosphere and $200 \mathrm{~m}$ in the troposphere, which are considered as thick turbulent layers. This simulation gave us confidence in the feasibility of the CAT detection 
using the proposed detection technique. This detectability limit is for a single lidar profile. In section 3 we describe the method of averaging the $F Q$ to increase the signal to noise ratio and enhance the level of detectability with a longer averaging time.

\section{INSTRUMENTAL SETUP AND DATA PROCESSING}

\section{A. Instrumental setup}

A CAT detection instrumental system has been added to the Rayleigh lidar installed at OHP. This lidar is part of the Network for Detection of Atmospheric Composition Changes (NDACC) station at $\mathrm{OHP}$ and measures routinely density and temperature profiles from 30 to $80 \mathrm{~km}$ [28]. A new optical reception system has been added to the Rayleigh lidar. It includes two channels, one receiving the signal with polarization parallel to the laser and the other the signal with perpendicular polarization. This allows detecting the presence of solid particles (cirrus clouds or aerosols) and prevents to use data contaminated by aerosol scattering. Water droplets do not depolarize. However when a liquid water cloud is present, the lidar signal increases substantially and it is straightforward to detect it and to discard the data for the CAT detection. We cannot totally excude the presence of a very weak layer of non-depolarizing aerosols. However we do not think that it may affect significantly the results because this weak layer has no reason to vary significantly within the time step of our analysis for turbulence detection (see discussion in Part 4B).

The schematic diagram for lidar transmitter and receiver is shown in Figure 2 and the main instrumental parameters are listed in Table 1. The master of the system is a frequency doubled Nd:YAG laser emitted $6 \mathrm{~m}$ off-axis of the receiving telescope to prevent high illumination of Photo-Multiplier Tubes (PMTs) by low altitude backscattering. The $532 \mathrm{~nm}$ output of the laser beam is sent to the sky with a $50 \mathrm{~Hz}$ pulse repetition rate, $7 \mathrm{~ns}$ pulse duration and a $15 \mathrm{~W}$ mean power. The beam divergence is reduced to $<0.1 \mathrm{mrad}$ using a $10 \mathrm{~cm}$ diameter beam expander.

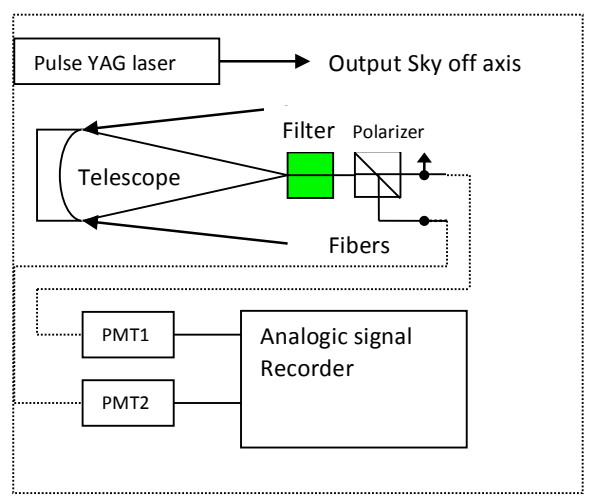

Figure 2: Optical layout of the Rayleigh lidar.

The received backscattered light is collected by a Newton telescope with $530 \mathrm{~mm}$ aperture (field of view $0.3 \mathrm{mrad}$ ), and then passes through a narrowband interference filter (bandwidth $1 \mathrm{~nm}$, peak transmission 61\%). As shown in the right of Figure 2, the collected light polarized components are split by the polarizer into two channels; approximately $99 \%$ of orthogonal component is detected directly by the photomultiplier tube R7205-01 PMT2 and 95\% of the parallel component by the PMT1. The two PMTs are used in analog mode due to the high intensity of the backscattered signal that may exceed $10^{9}$ photons/s at the maximum around 5-6 km altitude. The signal from the two channels is filtered (bandwidth $3.5 \mathrm{MHz}$ corresponding to $45 \mathrm{~m}$ vertical resolution) and digitized pulse to pulse by a dual channel $10 \mathrm{MHz}$ ( $15 \mathrm{~m}$ vertical sampling) constituted of 16 bits analog-to-digital converter (NI5922 board).

\section{B. Data processing}

Density fluctuations associated with turbulent layers are in the order of tenths of a percent and cannot be detected on a single density profile. In order to increase the signal to noise ratio the raw signal is first binned by 40 shots ( $0.8 \mathrm{~s}$ ), which provides 4500 vertical profiles during a one-hour sequence covering $45 \mathrm{~km}$ altitude range with $15 \mathrm{~m}$ vertical resolution. We obtain 4500 x 3000 matrices (one matrix per hour of measurement) representing the basic data set for this study. The background signal coming from the sky light and the noise detector is estimated from the signal at higher altitudes $(>30 \mathrm{~km})$ and is removed from the measured signal.

A 2D running average in vertical and in time is applied to the signal on $n_{t}$ time steps and $n_{z}$ altitude steps. In this study the parameters have been fixed to $n_{t}=25(20 \mathrm{~s})$ and $n_{z}=15(225 \mathrm{~m})$. It means that data are averaged in largely oversampled bins in both directions. These parameters are chosen to insure that we have enough signal in each averaged bin and to isolate the $F Q$ associated with structures of horizontal and vertical dimensions not larger than the expected external scale of atmospheric turbulence. Assuming a $10 \mathrm{~ms}^{-1}$ mean wind, the horizontal displacement of the sampled air mass is $200 \mathrm{~m}$ during the time integration, comparable with $150 \mathrm{~m}$ vertical integration. We obtain the matrix $S$ on which we compute the $F Q$ of atmospheric density in relative value $\overline{\left[(\Delta \rho / \rho)^{2}\right]}$ as a function of altitude. The $F Q$ is defined as the mean value of the square of the $2 \mathrm{D}$ signal fluctuations in vertical and in time using adjacent bins in both directions as illustrated in Figure 3 with the formulas:

$$
\Delta_{z}\left(t_{i,} Z_{j}\right)=\frac{S_{i, j}-\frac{1}{2}\left(S_{i, j-n z}+S_{i, j+n z}\right)}{\frac{1}{2} S_{i, j}+\frac{1}{4}\left(S_{i, j-n z}+S_{i, j+n z}\right)}
$$

$$
\begin{aligned}
& F Q\left(z_{j}\right)= \\
& \frac{1}{N_{t}} \sum_{i}\left[\Delta_{z}\left(t_{i}, z_{j}\right)-\frac{1}{2}\left(\Delta_{z}\left(t_{i-n t}, z_{j}\right)+\Delta_{z}\left(t_{i+n t}, z_{j}\right)\right)\right]^{2}
\end{aligned}
$$

where $N_{t}$ is the number of time elements on which we perform the summation. Figure 3 illustrates how data are averaged for parameters $n_{t}=3$ and $n_{z}=3$. In this example, the $2 \mathrm{D}$ fluctuation signal for $\left(t_{5}, z_{5}\right)$ is computed using the 9 bins around $S_{4,4}$.

We obtain one mean $F Q$ profile per hour on which we applied a moving vertical average on $n_{z}$ steps (red curves in Figure 4) to smooth the oscillations. This $F Q$ profile is the sum of the instrumental $F Q$ due to the detection noise and the atmospheric $F Q$ that we want to detect. It is not possible to estimate theoretically the instrumental $F Q$ with enough accuracy using only the characteristics of the lidar elements. We estimate it from the computation of the $F Q$ using a very short time integration $\left(n_{t}=1\right)$. If the statistic of the signal follows a Poisson law as expected for a lidar signal, the relative instrumental $F Q$ is inversely proportional to the lidar signal and therefore to the time integration and the atmospheric $F Q$ becomes negligible compared with the instrumental $F Q$.

The propagation of a laser beam through the atmosphere is also affected by the turbulence. This may cause some fluctuations in the lidar signal acquired on shot by shot basis. However this contribution is generally not taken into account in atmospheric lidar studies because the signal is rarely used at shot levels but integrated over tenths to thousands shots that reduces the effect inversely to the square root of the number of shots. Furthermore, we estimate the instrumental noise using lidar data acquired during a short integration time (nt=1 
corresponding to 40 shots) and if such a contribution exists, it will be included in this estimation.

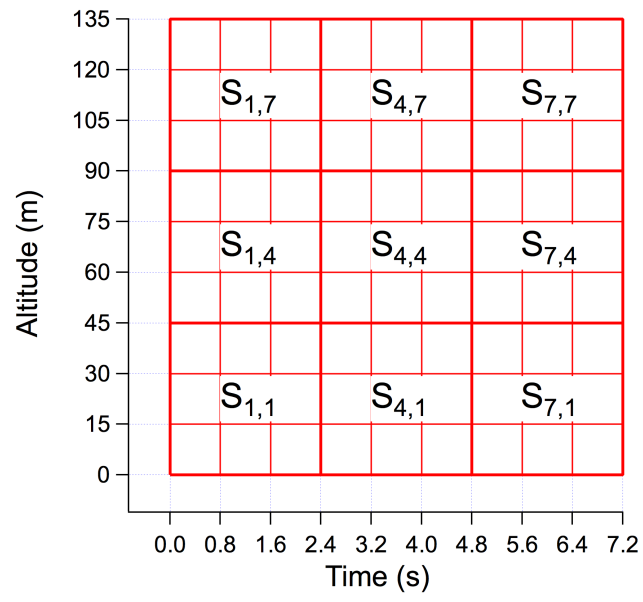

Figure 3: Schematic of the time and vertical signal averaging for $F Q$ computation. The elementary bins $(0.8 \mathrm{~s}, 15 \mathrm{~m})$ are represented with thin lines and the averaged bins for $n_{t}=3$ and $n_{z}=3$ with thick lines.

\section{LIKELY DETECTION OF TURBULENT LAYERS}

\section{A. Results}

After a careful screening of the data for the quality of the signal and the presence of clouds and aerosol layers, we were able to select only 3 one-hour sequences on December 8, 2008 and 2 one-hour sequences on June 23, 2009. Even if the meteorological conditions were not particularly favorable for the occurrence of strong CAT events, these data give us a good opprtunity to test the potential of our technique to detect moderate turbulent layers representative of the bakground state of the atmosphere.

Left side of Figures 4 and 5 represent the vertical profile of $F Q$ in logarithmic scale for the one-hour sequences and for the night average respectively on December 8, 2008 and on June 23, 2009. The blue and red thick curves represent respectively the estimated instrumental $F Q$ (computed with $n_{t}=1$ and divided by 25 to be normalized to $n_{t}=25$ ) and the total measured $F Q$. The atmospheric $F Q$ is expected to be the positive difference between the total and the instrumental $F Q s$ and is represented by the red shaded area between the blue and the red curves. We have to note that this difference includes the contribution of the measurement random noise around 0 . As indicated previously, the instrumental $F Q$ varies inversely to the lidar signal. It decreases with altitude below $5.5 \mathrm{~km}$ due to the progressive entrance of the lidar beam in the telescope field of view. Above $7 \mathrm{~km}$ the lidar beam is totally inside the telescope field of view and the $F Q$ increases with altitude due to the exponential decrease of the atmospheric density.
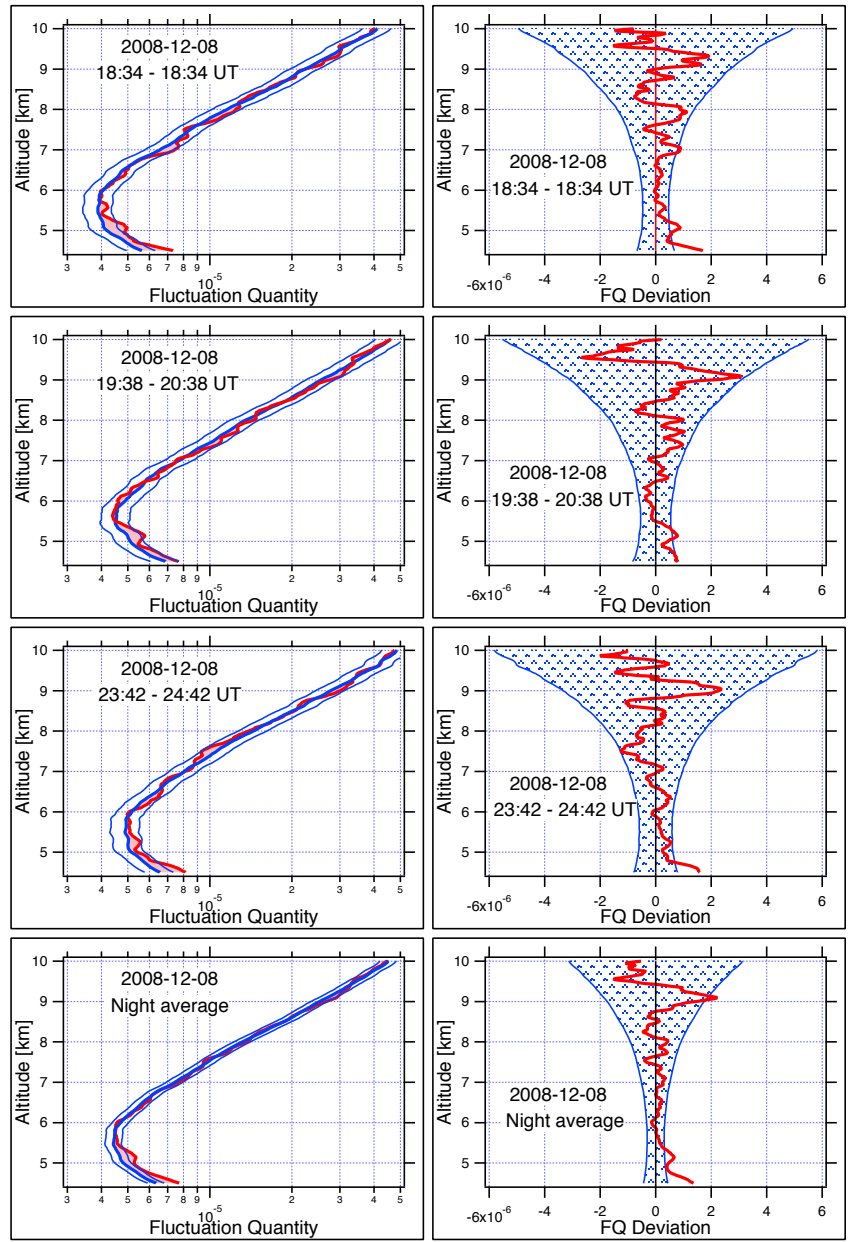

Figure 4: Left) Averaged $F Q$ (in relative value) with parameters $n_{t}=25$ and $n_{z}=15$ (red thick curve) and estimation of the instrumental $F Q$ (blue thick curve for the mean value and two blue light curves for the value $+/-2 \sigma$, ) for 3 sequences of one-hour integration time on December 08, 2008 and for the night average. The estimated atmospheric $F Q$ is the difference between the total observed $F Q$ and the estimated instrumental $F Q$ It is shaded in red. It includes the measurement random noise around 0 . Right) Difference beween the observed $F Q$ and the instrumental estimated $F Q$ (red) for the 3 one-hour periods and the night average. The blue shaded area corresponds to the $+/-2 \sigma$ uncertainty on the estimated instrumental $F Q$. The red curve on the right panel corresponds to the red shaded area on the left panel. 

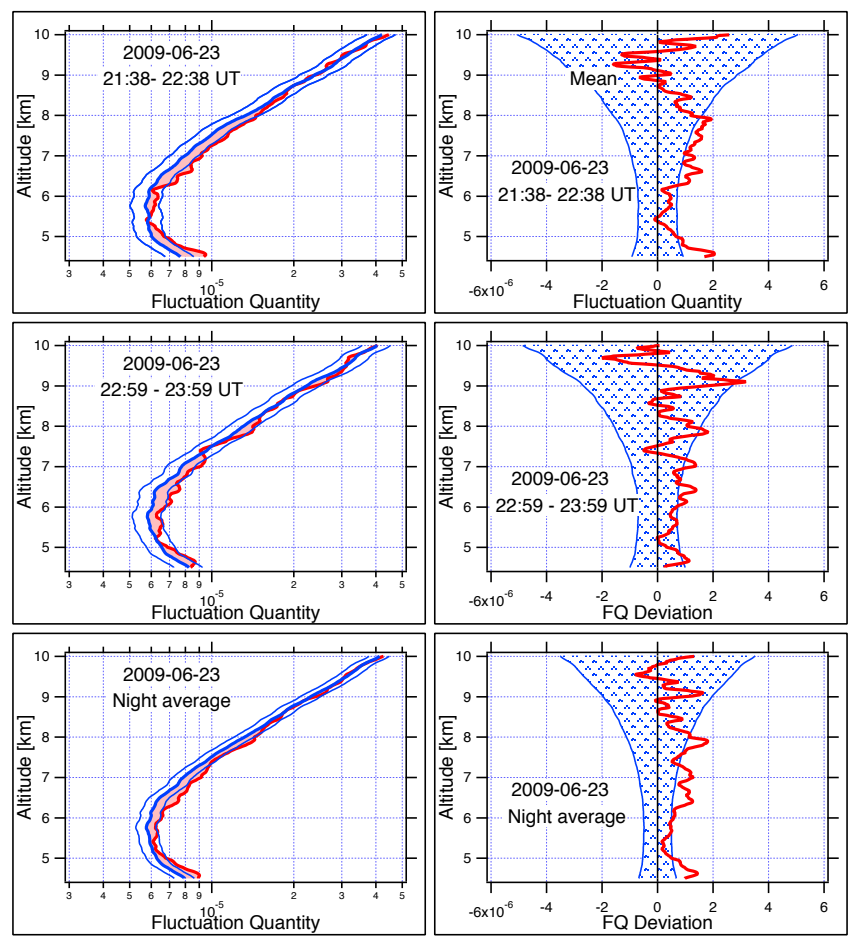

Figure 5: Same as Figure 4 for 2 one-hour sequences on June 23, 2009 and for the night average.

Due to the small amplitude of the difference between the observed and the estimated instrumental $F Q$, we have to perform a careful analysis of the instrumental noise uncertainty to be sure that the observed excess of fluctuations really originates in the atmosphere. We remark that the total $F Q$ exceeds the instrumental one by only a small fraction. In order to determine the significance of the observed $F Q$ excess, we have applied the same processing to one hour of simulated data. A $4500 \times 3000$ matrice of simulated lidar data has been generated with a Gaussian random noise generator added to a smooth simulated lidar signal (decreasing exponentially). Then we applied to the simulated signal the same processing than for the experimental signal. The standard deviation $(\sigma)$ of $F Q$ in the simulated lidar signal was found to be equal to $6 \%$ of its mean value. We consider that an excess of $F Q$ indicates a significant contribution of atmospheric $F Q$ at $95 \%$ confidence level if it exceeds the estimated instrumental $F Q$ by more than $12 \%$ ( $2 \sigma$ of its uncertainty ) for a one-hour sequence. For night time integration, this limit is decreased propartionaly to the square root of the number of sequences, 3 in December 2008 and 2 in June 2009 due to the statitics on more time bins.

In order to better visualize the excess of $F Q$ attributable to the atmospheric turbulence, we present in the right side of Figures 4 and 5 the difference between the observed total $F Q$ and the estimated instrumental $F Q$. The $+/-2 \sigma$ confidence interval is represented by the blue shaded area.

On December 8,2008 , the $+2 \sigma$ confidence interval is reached only in the lower part of the profile below $5 \mathrm{~km}$ on hourly sequences. We observe a peak of $F Q$ excess around $9 \mathrm{~km}$ that is at the limit of $2 \sigma$ during the second sequence and slightly above on the night average. We observe also a deficit of $F Q$ at some altitudes buy they are never significant at the $2 \sigma$ level.

On June 23, 2009, the average excess of $F Q$ is significantly larger and the $2 \sigma$ confidence interval is reached at several altitudes in the hourly sequences and in the night average. It is reached below $5 \mathrm{~km}$ and between 6.3 and $8 \mathrm{~km}$ during the first hour, in 5 narrow layers during the second hour and again below $5 \mathrm{~km}$ and between 6.3 and $8 \mathrm{~km}$ in the night average. We observe again that there is no layer with $F Q$ deficit reaching the $2 \sigma$ level.

Some negative peaks are close to the $-2 \sigma$ threshold. This is for instance the case during the $3^{\text {rd }}$ hour on December 08, 2008. This may happen due to random fluctuations of the signal. It is the reason why we should interpret with caution the positive peaks not exceeding $+2 \sigma$. The fact that more positive peaks exceed the $2 \sigma$ threshold with longer integration time, which is not the case for negative peaks that stay always below the threshold, gives us confidence in the reality of an atmospheric contribution to the observed fluctuations.

Another effect has to be considered as a possible perturbation in our analysis. The lidar signal may be slightly increased by the presence of very weak aerosol layers not detectable by the cross-polarizing channel. However we do not think that it may affect significantly the results. The main argument is that this weak layer has no reason to vary significantly within one $20 \mathrm{~s}$ time step of our analysis and its contribution to $F Q$ will be removed by the temporal differentiation in Equation 4.

In spite of their relatively small amplitude, positive excesses larger than $2 \sigma$ indicate the presence of turbulent layers. In order to confirm this finding, we processed the data of the stratosphere-troposphere (ST) radar setup at OHP, which was in operation on December 8, 2008. This is pulsed VHF radar, operating at $72 \mathrm{MHz}$ [29]. The antenna is a network of 16 coaxial-collinear antennas. Five antenna beams are used, one vertical and four oblique, $15^{\circ}$ off-zenith in two orthogonal planes. The back-scattered radio wave is coherently detected, a Doppler spectrum being calculated for each height range. Radar measurements consist of wind velocities and reflectivity in the $3-17 \mathrm{~km}$ range with a vertical resolution of $37 \mathrm{~m}$. The time resolution used here is 20 minutes.

The radar echoes, in clear-air conditions, are due to the small-scale inhomogeneities of the refraction index of air resulting from temperature and humidity fluctuations. For oblique beams (i.e. offzenith), the echo power depends on the intensity of the atmospheric turbulence. More precisely the radar reflectivity, defined as signal to noise ratio times the squared range, is proportional to the structure constant of refractive index $C_{n}^{2}$ averaged on the radar volume [30]. Figure 6 shows the time-height cross-section of radar reflectivity averaged on the four oblique beams observed on December 8-9, 2008. The radar shows the presence of enhanced refractivity below $5 \mathrm{~km}$ with increasing intensity during the night. This is in agreement with the observed layer with $F Q$ excess between 4.5 and $5 \mathrm{~km}$. The radar shows also a peak of refractivity around 8.6-9.2 km at 18:55 UT and a descending layer of enhanced refractivity from 9 to $7 \mathrm{~km}$ between 24:30 UT and 26:00 UT. This is again in rather good agreement with the lidar indicating a layer with $F Q$ excess peaking between 9 and $9.3 \mathrm{~km}$ during the 3 one-hour sequences, even if it is below the $2 \sigma$ confidence level. This comparison, even if it is not fully conclusive due to the low level of $F Q$ excess, reinforces our confidence in the reality of the lidar detection of turbulent layers.

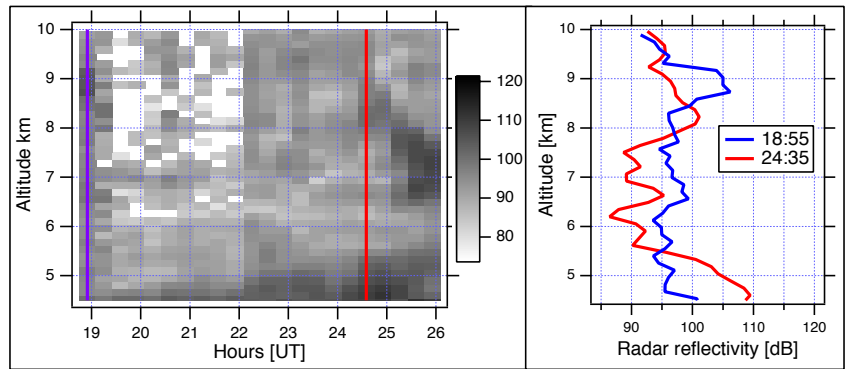


Figure 6: Left) Time-height section of the radar reflectivity (defined as signal to noise ratio times the squared range) in logarithm scale, arbitrary units. Due to a change of mode the level of the signal cannot be compared before 22UT and after 22UT. Right) Vertical profile of radar SNR at 18:55UT and $24: 35 \mathrm{UT}$ respectively during the $1^{\text {st }}$ and $3^{\text {rd }}$ lidar sequences. Times of the 2 vertical profiles are indicated by vertical blue on red lines in the left part.

In order to understand the reason for the larger atmospheric contribution to the observed $F Q$ excess on June 23, 2009 compared to December 8, 2008, we analyzed the meteorological situations for these two nights. Figure 7 presents the wind speed and direction during the two nights of lidar observations obtained at 00UT from the radiosounding profiles at Nimes (100 km West of OHP). The wind conditions were different during these 2 nights. On 8 to 9 December, 2008 OHP was located in an area of high pressure and a weak wind blew ( 2 to $10 \mathrm{~ms}^{-1}$ ) with changing direction with altitude from SouthEast at lower altitudes to South above $2 \mathrm{~km}$. These conditions were not favorable to the generation and the propagation of GWs and to the formation of turbulent layers. On June 23, 2009 a low pressure system was established on Adriatic Sea leading to North-East to North winds at all altitudes with up to $20 \mathrm{~ms}^{-1}$ at $1 \mathrm{~km}$ and about $10 \mathrm{~ms}^{-1}$ between 4 and $9 \mathrm{~km}$. Despite the similar magnitude of the wind in the altitude range of lidar data, the June conditions were more favorable to the generation and the upward propagation of GWs, with a $20 \mathrm{~ms}^{-1}$ low altitude wind blowing above the Alps North of the lidar station, and may explain why turbulent layers were more developed. We do not dispose of CAT probability forecast for these dates but the turbulent events that we have detected cannot be considered as strong events and are probably not predicted by the models. They are more representative of the background turbulence level always present in the atmosphere.
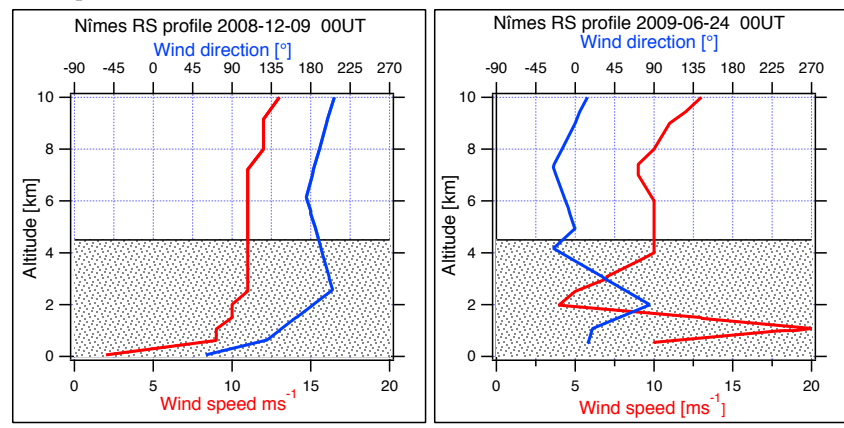

Figure 7: Wind speed (red, lower scale) and wind direction (blue, upper scale) form the Nîmes radiosounding on December 9, 2008 00:UT (left side) and June 24, 2009 00:UT (left side). The altitude range below the lidar data domain is grayed.

\section{B. Estimation of turbulent parameters}

In order to compare our results to published estimations of turbulent parameters obtained using other techniques, in particular stratosphere-troposphere (ST) radars [29, 31-32] it is necessary to translate them in more usual quantities like $C_{n}^{2}$ or $C_{T}^{2}$. By definition $C_{n}^{2}$ or $C_{T^{2}}$ are the mean square differences of air index of refraction and temperature at 1-meter distance in an isotropic turbulent layer (see [34] for more details). We assume that:

- we are well in the inertial range within the detected layers (scales smaller than external scale of turbulence);
- $\quad$ the computed variance $F Q$ represents correctly the square of relative fluctuations of atmospheric density and temperature for a distance equal to the vertical integration length $(225 \mathrm{~m})$;

- the Kolmogorov's $-2 / 3$ law for the structure function for isotropic turbulence applies.

This last hypothesis is discussed by [33] who showed that anisotropic turbulence should be assumed to interpret aircraft measurements of pressure wind and temperature at scales down to $300 \mathrm{~m}$. However the validity of this hypothesis increases when the scale decreases. The Kolmogorov two-third law applies for scales smaller than the external scale of turbulence, which corresponds roughly to the thickness of turbulent layers, typically $100-200 \mathrm{~m}$ in the troposphere [31]. The $225 \mathrm{~m}$ scale used in the present study, chosen not to small to not decrease too much the signal to noise ratio, is probably larger than the external scale for weak turbulent layers. This may cause an overestimation of turbulent parameters that we derive. However our goal is not to derive a very accurate value of turbulence parameters but to show that they are in the right order of magnitude.

Under these assumptions the two quantities $C_{n}^{2}$ or $C_{T}^{2}$ are related to the variance $F Q$ by the relations:

$$
\begin{aligned}
& C_{n}^{2}=225^{-2 / 3}(\boldsymbol{n}-\mathbf{1})^{2} F Q \\
& C_{T}^{2}=225^{-2 / 3} T^{2} F Q
\end{aligned}
$$

with the refractive index of air $n$ :

$$
n-1=0.776 .10^{-6} \frac{P}{T}
$$

where $P$ is the pressure in $\mathrm{Pa}$ and $T$ the temperature in $\mathrm{K}$.

This gives of course a crude estimate of turbulent parameters but with the good order of magnitude.

Turbulent parameters computed from the variance averaged in the altitude range 4.5 to $10 \mathrm{~km}$ with $n_{t}=25$ and $n_{z}=15$, assuming a mean temperature $T=240 \mathrm{~K}$ and a mean pressure $P=40000 \mathrm{~Pa}$, are given in Table 3. On June 23, 2009 the $F Q$ excess and the derived turbulent parameters are greater than on December 08, 2008 by almost a factor 4 . This is in agreement with the most favorable meteorological conditions for the occurrence of turbulent layers.

These values are realistic for the middle troposphere. They can be compared for instance with those published by [31] using ST radar data in the altitude range 11.5 to $15 \mathrm{~km}$ :

27/04/1998: $C_{n}^{2}=3.710^{-18}$ to $1.810^{-16}$, mean value $2.10^{-17} \mathrm{~m}^{-2 / 3}$

29/04/1998: $C_{n}^{2}=3.410^{-18}$ to $5.010^{-16}$, mean value $4.10^{-17} \mathrm{~m}^{-2 / 3}$

They can translate in $C_{T}^{2}$ assuming $P=16000 \mathrm{~Pa}$ and $T=220 \mathrm{~K}$ for this altitude range:

27/04/1998: $C_{T}{ }^{2}=$
$0.3010^{-3} \mathrm{~K}^{2} \mathrm{~m}^{-2 / 3}$

29/04/1998: $\quad C_{T}{ }^{2}=0.0510^{-3}$ to $7.610^{-3}$, mean value $0.6110^{-3} \mathrm{~K}^{2} \mathrm{~m}^{-2 / 3}$

These values are also in the same range than those estimated from ST radar measurements obtained at OHP with $C_{n}^{2}$ values ranging from $10^{-17}$ to $3 \times 10^{-16}$ for altitudes between 4 and $8 \mathrm{~km}$ [29]. In order to compare more quantitatively the results, Figure 8 represents the mean value, the range of observed values and the altitude range on which $C_{n}{ }^{2}$ is estimated for the two nights of lidar observations and the radar data published $[29,31]$. There is an overlap in the range of values observed by radar and lidar. Mean lidar values are larger than the radar ones. This is particularly true for the June 23, 2008 lidar sequence. This can be due to different atmospheric conditions, with a wind profile favorable to the propagation of GWs and the development of CAT during this particular night. The overestimation of turbulent parameters mentioned previously when the analysis scale is larger 
than the external scale of turbulence may also explain some part of the difference.

Table 3: Estimation of the turbulent parameters in the altitude range 4.5 to $10 \mathrm{~km}$ for the 5 one-hour-sequences and for the 2 night averages.

\begin{tabular}{|c|c|c|c|}
\hline Date and time & $F Q$ excess & $C_{n}^{2}\left(\mathrm{~m}^{-2 / 3}\right)$ & $C_{T^{2}}\left(\mathrm{~K}^{2} \cdot \mathrm{m}^{-2 / 3}\right)$ \\
\hline $\begin{array}{c}2008-12-08 \\
18: 34-19: 34\end{array}$ & $2.5610^{-7}$ & $1.1610^{-16}$ & $0.49^{0} 10^{-3}$ \\
\hline $\begin{array}{c}2008-12-08 \\
19: 38-20: 38\end{array}$ & $2.3810^{-7}$ & $1.0710^{-16}$ & $0.3710^{-3}$ \\
\hline $\begin{array}{c}2008-12-08 \\
23: 42-24: 42\end{array}$ & $0.4210^{-7}$ & $0.1910-16$ & $0.0710^{-3}$ \\
\hline $\begin{array}{c}2008-12-08 \\
\text { Night average }\end{array}$ & $1.7910^{-7}$ & $0.8110-16$ & $0.2810^{-3}$ \\
\hline $\begin{array}{c}2009-06-23 \\
21: 38-22: 38\end{array}$ & $7.4010^{-7}$ & $3.3510^{-16}$ & $1.1510^{-3}$ \\
\hline $\begin{array}{c}2009-06-23 \\
22: 59-23: 59\end{array}$ & $6.2110^{-7}$ & $2.8010^{-16}$ & $0.9710^{-3}$ \\
\hline $\begin{array}{c}2009-06-23 \\
\text { Night average }\end{array}$ & $6.7310^{-7}$ & $3.0410^{-16}$ & $1.0510^{-3}$ \\
\hline
\end{tabular}

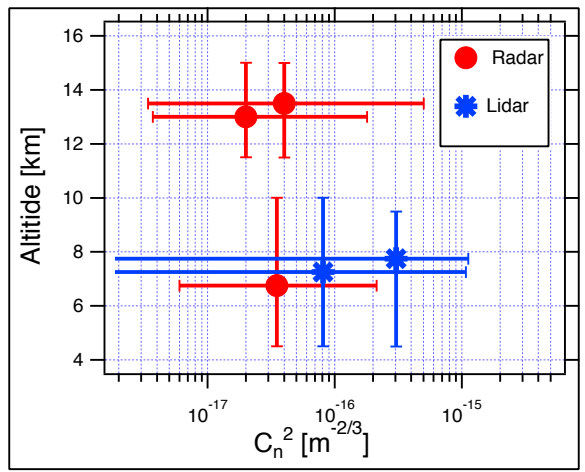

Figure 8: $C_{n}{ }^{2}$ estimated by lidar (this study, blue stars) and radar ([29,31], red full circles). The position of the marker indicates the mean value, vertical bars the altitude domain and horizontal bars the range of $C_{n}^{2}$ values within this altitude domain. For lidar the highest value is the nightly average on June 2009 and the lowest value the nightly average on December 2008. For radar the two observations at the highest altitudes have been made in April 1998 [31] and the observation at the lowest altitude in November 1998 [29].

\section{CONCLUSION}

A ground-based Rayleigh lidar was adapted and implemented to remotely detect the presence of turbulent layers in the upper troposphere. Field measurements were performed at OHP (France) in December 2008 and June 2009. The observed $F Q$ in bidimensional lidar signal showed excess compared to the estimated $F Q$ due to instrumental noise significant at the $95 \%$ confidence level in several altitude layers that can be attributed to CAT. The moderate level of CAT detected during these two nights is representative of the background atmospheric turbulence. During the first night, data from collocated ST radar were available. The altitude of the turbulent layers detected by the lidar was roughly consistent with that of layers with enhanced radar echo The derived values of turbulence parameters $C_{n}^{2}$ or $C_{T}^{2}$ are in the same range than those published in the literature using ST radar data. However the detection is at the limit of the instrumental noise and additional measurement campaigns with collocated ST radar measurements are highly desirable to confirm these initial results.
Furthermore the possible presence of weak non-depolarizing aerosol layers may play a minor role in the present results.

In spite of the small number of datasets available and the somewhat limited confidence level of CAT detection, we consider that our experiment proves the concept of detecting turbulent layers from the analysis of the fluctuations in a Rayleigh lidar signal. Possible improvements of a CAT detection system with a Rayleigh lidar would consist in the implementation of a high spectral resolution filter to discriminate between molecular and aerosol scattering and in the use of a laser with a higher repetition rate in the $\mathrm{kHz}$ range to improve the statistics of the signal without saturating the detector.

Despite these limitations, this is to our knowledge the first successful attempt to detect CAT in the free troposphere using a Rayleigh lidar system.

Funding Information. Agence Nationale de la Recherche (ANR) under contract number ANR06-BLAN-0398 MMEDTAC; European Community's Seventh Framework Programme (FP7/2007-2013) under grant agreement $n^{\circ} 233801$ DELICAT. 


\section{References}

1. Federal Aviation Administration, Turbulence staying safe, http://www.faa.gov/passengers/fly_safe/turbulence/, (2015).

2. A. P. Tvaryanas, "Epidemiology of turbulence-related injuries in airline cabin crew, 1992-2001," Aviat. Space. Environ. Med., vol. 74, no. 9, pp. 970-976, 2003

3. C. Cot, and Barat J., Wave-Turbulence Interaction in the Stratosphere: A Case Study, J. Geophys. Res., 91(D2), 2749-2756, (1986).

4. C. Cot, Equatorial messcale wind and temperature fluctuations in the lower atmosphere, J. Geophys. Res. 106(D2), 1523-1532, doi:10.1029/2000JD900597 (2001).

5. G.D. Nastrom, K.S. Gage, A climatology of atmospheric wavenumber spectra of wind and temperature observed by commercial aircraft, J. Atmos. Sci., 42, 950-960, (1985).

6. T. Tsuda, M. V. Ratnam, P. T. May, M. J. Alexander, R. A. Vincent, and A. MacKinnon, Characteristics of gravity waves with short vertical wavelengths observed with radiosonde and GPS occultation during DAWEX (Darwin Area Wave Experiment), J. Geophys. Res., 109, D20S03, doi:10.1029/2004JD004946. (2004).

7. Y. Murayama, T. Tsuda, and S. Fukao, Seasonal variation of gravity wave activity in the lower atmosphere observed with the MU radar, J. Geophys. Res., 99(D11), 23,057-23,069, doi:10.1029/94JD01717, (1994).

8. R. Wilson, A. Hauchecorne, and M.L. Chanin, Gravity wave spectra in the middle atmosphere as observed by Rayleigh lidar: Case studies, J. Geophys. Res. 96, 5153-5167 (1991).

9. R. Wilson, R, M.L. Chanin, M.L., and A. Hauchecorne, Gravity Waves in the Middle Atmosphere observed by Rayleigh Lidar: 2 Climatology, J.Geophys.Res., 96, 5169, (1991).

10. C.S. Gardner, M. S. Miller, and C. H. Liu, Rayleigh lidar observations of gravity wave activity in the upper stratosphere at Urbana, Illinois, J. Atmos. Sci. 46, 1838-1854, 1989.

11. J.A. Whiteway, and A.I. Carswell, Rayleigh lidar observations of thermal structure and gravity wave activity in the high Arctic during a stratospheric warming, J. Atmos. Sci. 51, 3122-3136 (1994).

12. T.E. VanZandt, A universal spectrum of buoyancy waves in the atmosphere, Geophys. Res. Lett., 9, 575-578 (1982).

13. C. Garrett, and W. Munk, Space-Time Scales of Internal Waves: A Progress Report, J. Geophys. Res., 80, 291-297 (1975).

14. T.N. Venkatesh, and J. Mathew, The problem of clear air turbulence: changing perspectives and understanding of the phenomenon, Sadhana, 38, 707-722, (2013.)

15. S.C. Tucker, S.C., W.A. Brewer, R.M. Banta, C.J. Senff, S.C. Sandberg, D.C. Law, A.M. Weickmann, and R.M. Hardesty, Doppler lidar estimation of mixing height using turbulence, shear and aerosol profiles, J. Atmos. Oceanic. Technol., 26, 673-688, (2009).

16. J.F. Barlow, T.M. Dunbar, E.G. Nemitz, C.R. Wood, M.W. Gallagher, F. Davies, E.O. O'Connor and R.M. Harrison, Boundary layer dynamics over London, UK, as observed using Doppler lidar during PEPARTEE-II, Atmos. Chem. Phys., 11, 2111-2125, (2011).

17. H. Inokushi, M. Furuta, and T. Inagaki, High altitude turbulence detection using an airborne Doppler lidar, Proceedings of 29th Congress of the International Council of the Aeronautical Sciences, St Petersburg, Russia, September 7-12 2014, (2014).

18. S.T. Shipley, D.H. Tracy, E.W. Eloranta, J.T. Trauger, J.T. Sroga, F.L. Roesler, and J.A. Weinman, Highspectral resolution lidar to measure optical scattering properties of atmospheric aerosols. 1: Theotry and instrumentation, Applied Optics, 22, 3716-3724, (1983).
19. J.W. Hair, C.A. Hostetler, A.L. Cook, D.B. Harper, R.A. Ferrare, T.L. Mack, W. Welch, L.R. Izquierdo, and F.E. Hovis, Airborne high spectral resolution lidar for profiling aerosol optical properties, Applied Optics, 47, 6734-6752, (2008).

20. D. Müller, C.A. Hostetler, R.A. Ferrare, S.P. Burton, E. Chemyakin, A. Kolgotin, J.W. Hair, A.L. Cook, D.B. Harper, R.R. Rogers, R.W. Hare, C.S. Cleckner, M.D. Obland, J. Tomlinson, L.K. Berg, and B. Schmid, Airborne multiwavelength High Spectral Resolution Lidar (HSRL-2) observations during TCAP 2012: vertical profiles of optical and microphysical properties of a smoke/urban haze plume over northeastern coast of the US, Atmos. Meas. Tech., 7, 3487-3496, (2014).

21. G. Baumgarten, Doppler Rayleigh/Mie/Raman lidar for wind and temperature measurements in the middle atmosphere up to 80 km, Atmos. Meas. Tech., 3, 1509-1518, (2010).

22. O. Reitebuch, C. Lemmerz, E. Nagel, and U. Paffrath, The airborne demonstrator for the direct-detection wind lidar ALADIN on ADM-Aeolus. Part I: instrument design and comparison to satellite instrument, J. Atmos. Oceanic. Technol., 26, 2501-2515, (2009).

23. P.J. Veerman, P. Vrancken, and L. Lombard, Flight testing DELICAT - A promise for medium-range clear air turbulence detection, Proceedings of European 46th SETP and 25th SFTE Symposium, 15-18 June 2014, Luleå, Sweden, (2014).

24. A.S. Gurvich, Lidar sounding of turbulence based on the backscatter enhancement effect, Izvestiya Atmospheric and Oceanic Physics, 48, 585-594, (2012).

25. A.N. Kolmogorov, A.N. The local structure of turbulence in incompressible viscous fluids at very large Reynolds numbers, Dokl. Akad. Nauk. SSSR 30, 299-303. Reprinted in Proc. R. Soc. London A 434, 9-13 (1991), (1941).

26. A. Hauchecorne, and M.L. Chanin, Density and temperature profiles obtained by lidar between 35 and 70 km, Geophys. Res. Lett. 7, 565-568 (1980).

27. V. I. Tatarski, Wave propagation in a turbulent media, translated by R.A. Silverman. Mc Graw Hill, New York, 285pp., 1961.

28. P. Keckhut, A. Hauchecorne and M.L. Chanin, A critical review of the data base acquired for the long term surveillance of the middle atmosphere by Rayleigh lidar, J. Atm. Ocean. Tech. 10, 850-867 (1993).

29. J. Dole, and R. Wilson, Estimates of turbulent parameters in the lower stratosphere-upper troposphere by radar observations: a novel twist , Geophys. Res. Lett., 27, 2625-2628, (2000).

30. H. Ottersten, Radar backscattering from the turbulent clear atmosphere, Radio Sci., 4, 1251-1255, 1969.

31. J. Dole, R. Wilson, F. Dalaudier and C. Sidi, Energetics of Small scale turbulence in the lower stratosphere from High resolution radar measurements, Annales Geophysicae, 19, 945-952, (2001).

32. R. Wilson, F. Dalaudier and F. Bertin,, Estimation of the turbulent heat flux in the lower stratosphere from high resolution radar measurements, Geophys. Res. Lett., 32, L21811 (2005).S. Lovejoy, A. F. Tuck, D. Schertzer, and S. J. Hovd, Reinterpreting aircraft measurements in anisotropic scaling turbulence, Atmos. Chem. Phys., 9, 5007-5025 (2009).

33. S. Lovejoy, A. F. Tuck, D. Schertzer, and S. J. Hovd, Reinterpreting aircraft measurements in anisotropic scaling turbulence, Atmos. Chem. Phys., 9, 5007-5025 (2009).

34. K.S. Gage, J.L. Green, and T.E. Van Zandt, Use of Doppler radar for the measurement of atmospheric turbulence parameters form the intensity of clear-air echoes, Radio Science, 15, 407-416 (1980). 